\title{
Role of tumor microenvironment in the efficacy of BCG therapy
}

\author{
Omar M Ibrahim¹, Ravindra K Pandey², Gurkamal Chatta ${ }^{3}$ and Pawel Kalinski* \\ ${ }^{1}$ Department of Medicine, Division of Translational Research, Roswell Park Comprehensive Cancer Center, Buffalo, NY, United States \\ ${ }^{2}$ Department of Cell Stress Biology, Roswell Park Comprehensive Cancer Center, Buffalo, NY, United States \\ ${ }^{3}$ Department of Urology, Roswell Park Comprehensive Cancer Center, Buffalo, NY, United States
}

\begin{abstract}
Despite its significant overall efficacy, BCG fails to benefit a substantial proportion of bladder cancer (B1Ca) patients. Here, we review recent data highlighting the role of tumor microenvironment (TME) in limiting antitumoral activity of BCG treatment and emerging opportunities to target TME to enhance the overall outcomes in BCG-treated B1Ca patients.
\end{abstract}

The presence of high levels of cytotoxic T cells (CTLs) and low levels of myeloid-derived suppressor cells (MDSCs) and regulatory T cells (Tregs) is associated with improved clinical outcomes in bladder cancer (BlCa) patients [1-4]. These observations highlight the importance of developing new strategies to rebalance $\mathrm{BlCa}$ tumor microenvironment (TME) to enhance local immune surveillance and enhance treatment outcomes.

BCG, which is known to activate the immune system via Tolllike receptor (TLR)-2, 4 \& 9 signaling, is the oldest immunotherapy effectively used for treatment of non-muscle invasive bladder cancer (NMIBC) [5,6]. However, 31-78\% of BCG-treated patients suffer from bladder cancer recurrence and $45 \%$ progress to muscle invasive bladder cancer (MIBC) [7]. In order to control the progression of BCG-nonresponsive NMIBC, radical cystectomy is usually performed, which negatively affects quality of life [7]. Therefore, improved treatments are needed to allow bladder preservation.

Tumor-infiltrating immune cells are comprised of immunostimulatory and suppressive populations, with antitumor or tumor-promoting functions [2]. High local numbers of CD8 ${ }^{+}$CTLs are associated with improved prognosis in $\mathrm{BlCa}$ and other tumors $[1,8,9]$. The presence of intratumoral CTLs is critically important for the clinical activity of immune checkpoint inhibition (ICI) with PD-1, PD-L1 or CTLA-4 blocking antibodies [10-12]. In contrast, preferential accumulation of regulatory $\mathrm{T}$ cells (Tregs) and myeloid-derived suppressor cells (MDSCs) predicts poor survival $[13,14]$. MDSCs work via multiple pathways including their production of inflammatory mediators: Indoleamine 2,3-Dioxygenase 1 (IDO1), Interleukin 10 (IL10), Nitric Oxide Synthase 2 (NOS2) and Arginase 1 (ARG1) that suppress proliferation of $\mathrm{CD}^{+} \mathrm{T}$ cells and their development into Granzyme $\mathrm{B}^{+} / \mathrm{CD}^{+}$effector/killer T cells (CTLs) [15].

CTLs and suppressive cells are attracted to tumor tissues by different sets of chemokines [16]. CCL5/RANTES, a ligand for CCR5, and CXCL9/MIG and CXCL10/IP10, both CXCR3 ligands (Figure 1a), enhance antitumor immunity, by recruiting Th1, CTL and NK cells to the TME. In contrast, Tregs, neutrophils, MDSCs and type -2 (M2) macrophages promote tumor growth $[16,17]$. Their attraction to TME is mediated by CXCR1 and CXCR2 ligand, CXCL8/IL8, CXCR4 ligand, CXCL12/SDF1, and CCR4 ligand, CCL22/MDC. CXCL8 and CXCL12 preferentially recruit MDSCs, while CCL22 preferentially recruits Tregs (Figure 1b) [18], which both promote tumor growth [17,19].

Our recent study identified the induction of the MDSC- and Tregattractants, CCL22 and CXCL8, as the undesirable side effect of BCG in human $\mathrm{BlCa}$ tissues [20], raising the possibility that the modulation of the chemokine system can be used to enhance the effectiveness of BCG therapies and counteract BCG-unresponsiveness. Since IFNa promotes antitumor immunity in many BlCa models [21], combination of BCG and IFNa has been introduced into clinical testing [22], but failed to demonstrate any advantage over BCG alone in NMIBC relapsed patients $[23,24]$. These disappointing results may be explained by the observations that the combination of IFNa with BCG (TLR-2, $4 \& 9$ activator), alone or with poly-IC (TLR-3 agonist) enhances not only CXCL10 production but also CCL22 production in human $\mathrm{BlCa}$ tissues [20]. Another example of promising combinatorial approaches is the addition of photodynamic therapy (PDT) to BCG, which recently showed to induce $50 \%$ responsiveness in BlCa patients, however at a cost of $80 \%$ cardiotoxicity [25]. Local instillation of photosensitizer for the treatment of recurrent $\mathrm{BlCa}$ in conjunction with BCG-therapy has shown improved response, an approximate of $71 \%$ in the 2-year followup. This outcome was superior when compared with transurethral resection of bladder tumor (TURBT) followed by either BCG therapy or PDT [26]. In general, the toxicity in PDT treatment depends on the photosensitizer used, and the combination approach with the use of tumor-selective PDT agent represents a particularly promising therapeutic approach [27].

${ }^{\star}$ Correspondence to: Pawel Kalinski, MD, PhD, Roswell Park Cancer Center, 945 CSC Building, Elm \& Carlton Streets, Buffalo, NY 14263, USA, E-mail: Pawel.Kalinski@RoswellPark.org

Key words: chemokines, immunomodulation, immune dysfunction, tumor microenvironment, bladder cancer, Bacillus Calmette-Guérin (BCG), immunotherapy

Received: July 27, 2020; Accepted: August 11, 2020; Published: August 17, 2020 
A
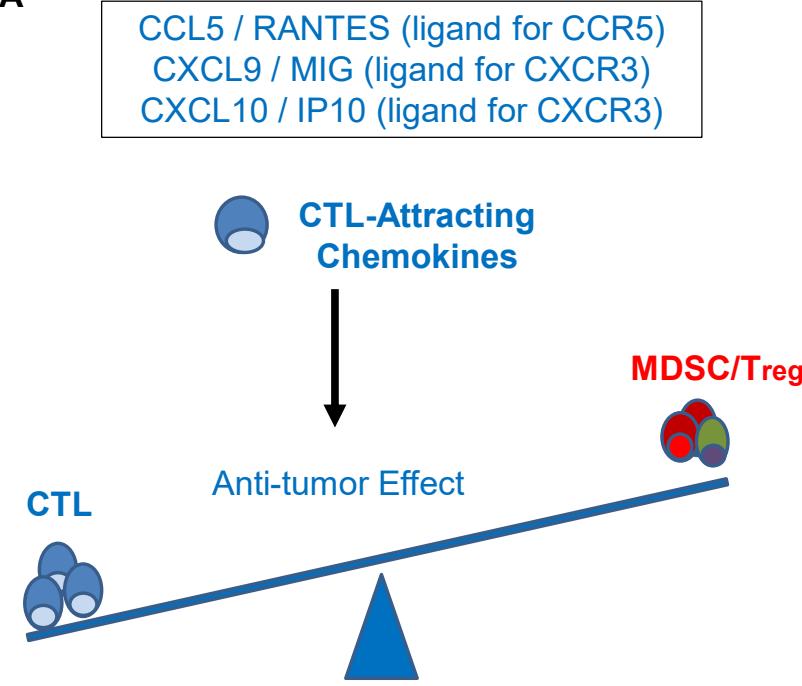

B

\section{CXCL8 / IL8 (ligand for CXCR1, CXCR2) \\ CXCL12 / SDF1 (ligand for CXCR4) \\ CCL22 / MDC (ligand for CCR4)}

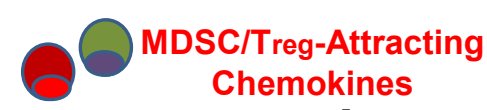

Chemokines

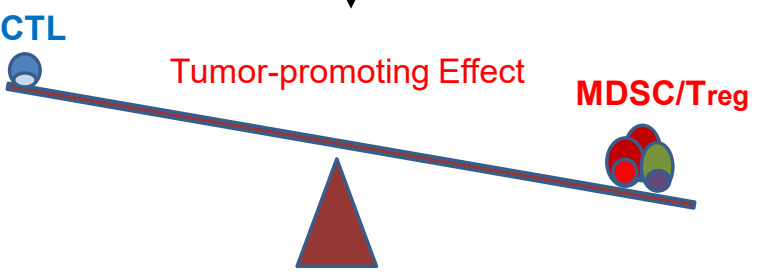

Figure 1. Role of CTLs/NK/Th1-attracting and Treg/MDSCs attracting chemokines in the tumor microenvironment resistance and progression. A. CCL5/RANTES (a ligand for CCR5 receptor) and CXCL9/MIG and CXCL10/IP10 (ligands for CXCR3 receptor) preferentially attract antitumor effector cells, such as CTLs, Th1 and NK cells, and additional DCs, to promote local antitumor immunity in BlCa TME. B. In contrast, CXCL8/IL8 (CXCR1, CXCR2 ligand), CXCL12/SDF1 (CXCR4 ligand) and CCL22/MDC (CCR4 ligand) attract Tregs and MDSCs, which promote local immune suppression, resistance to immune attack and tumor growth

BCG-triggered local inflammation is reflected by the increased numbers of macrophages, T cells, B cells, NK cells and neutrophils in the urine of BlCa patients after BCG administration [28]. High levels of such cells predict the clinical response to ICI blockers combined with BCG [29]. On the other hand, elevated local (tumor tissue and urine) recruitment of Tregs after BCG administration predicts poor treatment outcomes [2,30]. Reduced recurrence-free survival has been also reported in BlCa patients whose local MDSCs dominated T cells after BCG administration [4]. The negative impact of Tregs and MDSCs is in sharp contrast to $\mathrm{CD} 8^{+} \mathrm{T}$ cells, which predict better survival of $\mathrm{BlCa}$ patients [1], and may help to identify the $25 \%$ of BCG-non-responsive patients with enhanced $\mathrm{PD}-\mathrm{L} 1^{+} \mathrm{T}$ cells influx will benefit from the combination with ICI [29].

\section{Conclusions}

These observations indicate that the balance between the chemokines which selectively attract $\mathrm{CCR}^{+} / \mathrm{CXCR}^{+}$antitumor effector cells (CTL/NK) versus tumor promoting suppressive cells (MDSC/Treg) may regulate the effectiveness of BCG and other forms of immunotherapy, and may be targeted to enhance the clinical outcomes. The resulting treatments may boost BCG activity against NMIBC in terms of efficacy and durability and, potentially, obviate the need for a cystectomy. The key questions are: What molecular pathways regulate the antitumor and tumor promoting aspects of BCG activity in $\mathrm{BlCa}$ TME and whether can they be selectively manipulated to enhance the magnitude and selectivity of action of the modulation of TME?

\section{Declarations}

Competing interests: The authors declare no financial conflict of interest.

Financial support: This work was supported by the NIH/NCI grant 1P01CA234212 and the Rustum Family Foundation (to PK) and by the Fulbright US Exchange Award AY2018-2019 (to OMI).

\section{References}

1. Sharma P, Shen Y, Wen S, Yamada S, Jungbluth AA, et al. (2007) CD8 tumor-infiltrating lymphocytes are predictive of survival in muscle-invasive urothelial carcinoma. Proc Natl Acad Sci U S A 104: 3967-3972. [Crossref]

2. Pichler R, Fritz J, Zavadil C, Schafer G, Culig Z, et al. (2016) Tumor-infiltrating immune cell subpopulations influence the oncologic outcome after intravesical Bacillus Calmette-Guerin therapy in bladder cancer. Oncotarget 7: 39916-39930. [Crossref]

3. Chevalier MF, Schneider AK, Cesson V, Dartiguenave F, Lucca I, et al. (2018) Conventional and PD-L1-expressing regulatory $\mathrm{T}$ cells are enriched during BCG therapy and may limit its efficacy. Eur Urol 74: 540-544. [Crossref]

4. Chevalier MF, Trabanelli S, Racle J, Salome B, Cesson V, et al. (2017) ILC2-modulated $\mathrm{T}$ cell-to-MDSC balance is associated with bladder cancer recurrence. J Clin Invest 127: 2916-2929. [Crossref]

5. Heldwein KA, Liang MD, Andresen TK, Thomas KE, Marty AM, et al. (2003) TLR2 and TLR4 serve distinct roles in the host immune response against Mycobacterium bovis BCG. J Leukoc Biol 74: 277-286. [Crossref]

6. von Meyenn F, Schaefer M, Weighardt H, Bauer S, Kirschning CJ, et al. (2006) Toll-like receptor 9 contributes to recognition of Mycobacterium bovis Bacillus Calmette-Guerin by Flt3-ligand generated dendritic cells. Immunobiology 211: 557-565. [Crossref]

7. Pettenati C, Ingersoll MA (2018) Mechanisms of BCG immunotherapy and its outlook for bladder cancer. Nat Rev Urol 15: 615-625. [Crossref]

8. Sato E, Olson SH, Ahn J, Bundy B, Nishikawa H, et al. (2005) Intraepithelial CD8+ tumor-infiltrating lymphocytes and a high $\mathrm{CD} 8+$ /regulatory $\mathrm{T}$ cell ratio are associated with favorable prognosis in ovarian cancer. Proc Natl Acad Sci U S A 102: 1853818543. [Crossref]

9. Chen Z, Chen X, Zhou E, Chen G, Qian K, et al. (2014) Intratumoral CD8(+) cytotoxic lymphocyte is a favorable prognostic marker in node-negative breast cancer. PLoS One 9: e95475. [Crossref]

10. Herbst RS, Soria JC, Kowanetz M, Fine GD, Hamid O, et al. (2014) Predictive correlates of response to the anti-PD-L1 antibody MPDL3280A in cancer patients. Nature 515: 563-567. [Crossref]

11. Topalian SL, Hodi FS, Brahmer JR, Gettinger SN, Smith DC, et al. (2012) Safety, activity, and immune correlates of anti-PD-1 antibody in cancer. $N$ Engl J Med 366: 2443-2454. [Crossref]

12. Friese C, Harbst K, Borch TH, Westergaard MCW, Pedersen M, et al. (2020) CTLA-4 blockade boosts the expansion of tumor-reactive CD8+ tumor-infiltrating lymphocytes in ovarian cancer. Scientific Reports 2020; 10: 3914. 
13. Winerdal ME, Marits P, Winerdal M, Hasan M, Rosenblatt R, et al. (2011) FOXP3 and survival in urinary bladder cancer. BJU Int 2011; 108: 1672-1678

14. Bergenfelz C, Larsson AM, von Stedingk K, Gruvberger-Saal S, Aaltonen K, et al (2015) Systemic Monocytic-MDSCs are generated from monocytes and correlate with disease progression in breast cancer patients. PloS one 10: e127028. [Crossref]

15. Kalinski P (2012) Regulation of immune responses by prostaglandin E2. J Immunol 188: 21-28. [Crossref]

16. Griffith JW, Sokol CL, Luster AD (2014) Chemokines and chemokine receptors: positioning cells for host defense and immunity. Annu Rev Immunol 32: 659-702. [Crossref]

17. Franciszkiewicz K, Boissonnas A, Boutet M, Combadiere C, Mami-Chouaib F (2012) Role of chemokines and chemokine receptors in shaping the effector phase of the antitumor immune response. Cancer Res 72: 6325-6332. [Crossref]

18. Sokol CL, Luster AD (2015) The chemokine system in innate immunity. Cold Spring Harb Perspect Biol 7: a016303. [Crossref]

19. Vilgelm AE, Richmond A (2019) Chemokines modulate immune surveillance in tumorigenesis, metastasis, and response to immunotherapy. Front Immunol 10: 333. [Crossref]

20. Muthuswamy R, Wang L, Pitteroff J, Gingrich JR, Kalinski P (2015) Combination of IFNalpha and poly-I:C reprograms bladder cancer microenvironment for enhanced CTL attraction. J Immunother Cancer 3: 6. [Crossref]

21. Papageorgiou A, Lashinger L, Millikan R, Grossman HB, Benedict W, et al. (2004) Role of tumor necrosis factor-related apoptosis-inducing ligand in interferon-induce apoptosis in human bladder cancer cells. Cancer Res 2004; 64: 8973-8979.
22. Joudi FN, Smith BJ, O'Donnell MA (2006) Final results from a national multicenter phase II trial of combination bacillus Calmette-Guerin plus interferon alpha-2B for reducing recurrence of superficial bladder cancer. Urol Oncol 24: 344-348. [Crossref]

23. Nepple KG, Lightfoot AJ, Rosevear HM, O'Donnell MA, Lamm DL (2010) Bacillus Calmette-Guerin with or without interferon alpha- $2 b$ and megadose versus recommended daily allowance vitamins during induction and maintenance intravesical treatment of nonmuscle invasive bladder cancer. J Urol 184: 1915-1919. [Crossref]

24. Lamm D, Brausi M, O'Donnell MA, Witjes JA (2014) Interferon alfa in the treatment paradigm for non-muscle-invasive bladder cancer. Urol Oncol 32: 35.e21-30. [Crossref]

25. Waidelich R, Stepp H, Baumgartner R, Weninger E, Hofstetter A, et al. (2001) Clinical experience with 5 -aminolevulinic acid and photodynamic therapy for refractory superficial bladder cancer. J Urol 165: 1904-1907. [Crossref]

26. Szygula M, Pietrusa A, Adamek M, Wojciechowski B, Kawczyk-Krupka A, et al. (2004) Combined treatment of urinary bladder cancer with the use of photodynamic therapy (PDT) and subsequent BCG-therapy: a pilot study. Photodiagnosis and Photodynamic Therapy 1: 241-246.

27. Railkar R, Agarwal PK (2018) Photodynamic therapy in the treatment of bladder cancer: Past challenges and current innovations. Eur Urol Focus 4: 509-511. [Crossref]

28. Fuge O, Vasdev N, Allchorne P, Green JS (2015) Immunotherapy for bladder cancer Research and Reports in Urology 2015; 7: 65-79.

29. Kates M, Matoso A, Choi W, Baras AS, Daniels MJ, et al. (2020) Adaptive immune resistance to intravesical BCG in non-muscle invasive bladder cancer: Implications for prospective BCG-unresponsive trials. Clin Cancer Res 26: 882-891. [Crossref]

30. Fenner A (2018) BCG enriches Treg cells. Nat Rev Urol 15: 591.

Copyright: $@ 2020$ Ibrahim OM. This is an open-access article distributed under the terms of the Creative Commons Attribution License, which permits unrestricted use, distribution, and reproduction in any medium, provided the original author and source are credited. 